\title{
La Prise En Charge Des Detenus Infectes Par Le Vih/Sida A La Maison D'arret Et Correction D'abidjan (Maca)
}

\author{
Alexandre Kouassi Ahissan \\ Docteur en Criminologie, Psycho-criminologue, \\ UFR Criminologie Université Félix Houphouët Boigny de Cocody
}

doi: 10.19044/esj.2017.v13n27p342 URL:http://dx.doi.org/10.19044/esj.2017.v13n27p342

\begin{abstract}
This article shows the effects of different forms of care on the living conditions of prisoners infected with HIV / AIDS. From a methodological point of view, the study used three instruments: direct observation, individual free interview and documentary research. As for the method of data analysis, content analysis and qualitative analysis were used to better understand the content of the respondents' speeches. This study presents the various forms of care provided to prisoners living with HIV / AIDS, which begin with awareness-raising and voluntary testing. And these care are often contributed by their living conditions.
\end{abstract}

Keywords: Care, detained, infected hiv / aids

\section{Résumé}

Cet article montre les effets des différentes formes de prises en charge sur les conditions de vie des détenus infectés par le VIH/Sida. Du point de vue méthodologique, l'étude a eu recours à trois instruments à savoir l'observation directe, l'entretien libre individuel et la recherche documentaire. Quant à la méthode d'analyse des données, l'analyse de contenu et l'analyse qualitative ont été utilisées pour mieux comprendre le contenu des discours des enquêtés. Cette étude présente les différentes formes de prises en charge bénéficiées par les prisonniers vivant avec le $\mathrm{VIH} /$ Sida qui commencent par la sensibilisation et le dépistage volontaire. Et ces prises en charge sont souvent mises en contribution par leurs conditions de vie.

Mots clés : Prise en charge, détenu, infecté vih/sida 


\section{Introduction:}

\section{Quelques repères théoriques}

$\mathrm{La}$ prise en charge des détenus infectés par le VIH/Sida est une préoccupation des nombreux professionnels, praticiens et chercheurs qui ont décidé à intégrer dans leurs réflexions et pratiques.

Ainsi lors de la réunion internationale sur la santé en prison et la santé publique qui s'est tenue à MOSCOU les 23 et 24 octobre 2003 à l'initiative de l'organisation mondiale de la santé et la fédération de RUSSIE, les délégués ont fondé leurs discussions sur les normes internationales fondamentales relatives à la nécessité d'établir un lien étroit entre la santé publique et les soins de santé dispensés aux détenus (Déclaration de MOSCOU, 2003).

Selon Ousmane et al(2015), les conditions d'incarcération, les pratiques homosexuelles et l'absence de préservatifs dans les prisons aggravent la vulnérabilité des détenus face au VIH/Sida. La mise en place d'un programme de prévention et de prise en charge des patients VIH permettraient de réduire de manière significative le risque de transmission du VIH en prison.

Les conditions de détention dans les prisons en Afrique représentent une menace pour la vie et la santé des personnes incarcérées et de la société dans son ensemble. Les taux de mobilité et de mortalité sont élevés, les conditions sanitaires des prisons sont extrêmement dégradées.

Les ressources affectées aux prisons par les gouvernements sont réduites, en particulier pour l'amélioration de la santé et la formation des personnels. La Cote d'Ivoire n'échappe pas à cette règle. La santé des populations carcérales pose un problème de santé publique qui mérite une réponse spécifique et multisectorielle.

Pour mieux cerner ce travail, deux concepts qui paraissent essentiels à définir sont : « détenus infectés par le VIH/Sida » et « prise en charge ».

D'abord il faut définir le concept « détenus infectés par le VIH/Sida » mais avant, il nous parait judicieux de faire un éclairage sur la notion de « détenu ».

En effet, selon Le Grand Robert de la langue française(2005), le détenu est celui là même qui est maintenu en captivité, coupable criminel. C'est un criminel détenu en prison. C'est aussi un prisonnier, un bagnard, un forçat.

Pour GOBA et ZADY (2017), un détenu est un individu qui est en prison, incarcéré en tant que prévenu, non encore jugé ou en tant que condamné qui purge sa peine après jugement.

Le détenu, se définit conformément au décret 69-189du 14mai 1969,portant réglementation des établissements pénitentiaires et fixant les modalités d'exécution des peines privatives de liberté et au regard des articles 63,76 et 154 du code procédure pénale ivoirien ou de l'article 9 de la 
loi 63-1 du 11 janvier 1963 comme toute personne faisant l'objet d'une mesure privative de liberté à l'exclusion de celles gardées à vue.

Les détenus comprennent les condamnés, les prévenus et les contraignables par corps. (ZADY, 2014).

Pour GUERIN(2003), la population carcérale est formée de prévenus (personnes détenues qui n'ont pas encore été jugées ou dont la condamnation n'est pas définitive) et de condamnés(personnes détenues en vertu d'une condamnation judiciaire définitive).

Apres avoir défini la notion « détenu », nous allons voir ce qu'il en est pour le concept «détenus infectés par le VIH/Sida». Il s'agit des prisonniers infectés par le virus VIH/Sida. Selon (Ahissan, 2010), on dit qu'une personne est infectée par le VIH lors qu'elle est séropositive. Les détenus infectés par le VIH/Sida ou séropositifs sont non seulement des porteurs sains qui ne présentent aucun signe physique mais aussi les malades avérés c'est-à-dire ceux qui présentent des signes physiques de la maladie.

Pour l'OMS (2001), les prisons abritent un grand nombre de personnes infectées par le VIH et à risque, et pourtant les détenus constituent l'un des groupes de populations les moins pris en compte dans les plans nationaux de lutte contre le VIH. Pourtant les conditions dans les établissements pénitentiaires sont particulièrement favorables à la transmission de nombreuses maladies infectieuses. La surpopulation et les conditions insalubres dans les établissements pénitentiaires, où la consommation de drogues injectables et les rapports sexuels non protégés sont des pratiques courantes, en font un terrain idéal pour la transmission du VIH. (Onusida, 2007).

La majorité des détenus sont des hommes notamment en Afrique. De ce fait, le milieu carcéral est particulièrement propice à la violence et aux rapports homosexuels. Les rapports hétérosexuels et homosexuels en prisonconsensuels ou forcés-jouent un rôle moteur dans la propagation de l'infection(Curtis, 2004).

Selon l'Onusida (2006), l'Afrique subsaharienne est la région la plus durement touchée par l'épidémie, avec près des deux tiers du nombre total de séropositifs dans le monde.

Le VIH en milieu carcéral est à la fois un problème de santé publique et une question de droit de l'homme qu'il convient de traiter d'urgence en vue d'une prise en charge efficace sur le continent. Pourtant et malgré l'augmentation sensible des ressources consacrées à la lutte contre l'épidémie au niveau national et international, les prisons en Afrique subsaharienne n'ont guère reçu d'attention (Onusida, 2007).

En ce qui concerne la Côte d'Ivoire précisément à Abidjan, la maison d'arrêt et de correction a une prévalence moyenne chez les détenus de 5,88\% repartie de manière suivante : chez les hommes $(5,39 \%)$ et chez les femmes 
(16,6\%). Cette prévalence est le double de celle de la population externe. Pour une population assez touchée par le VIH, quelle est la prise en charge adaptée ? Donc pour avoir une idée de la prise en charge de détenus, il parait opportun de définir le concept « prise en charge ». En effet la prise en charge des détenus infectés par le VIH/Sida est très difficile. Ainsi selon Bedell (2001), le diagnostic et le traitement sont souvent complexes. Pour cette raison, les soins devraient être apportés par des médecins spécialisés dans le $\mathrm{VIH} / \mathrm{Sida}$, et des accords devraient être passés avec des centres régionaux de prévention et de traitement du Sida pour faciliter l'accès des prisonniers atteints du VIH/Sida au meilleur traitement possible. Ce qui n'est pas toujours le cas. Ainsi, selon Onusida (2007), il est tres rare que les détenus aient accès aux tests de dépistage du VIH ou de la tuberculose, et encore moins aux traitements antirétroviraux contre le VIH. Pour ceux qui ont la chance d'être dépistés séropositifs, la prise en charge devient problématique.

Les soins de santé dans la plupart des prisons africaines sont généralement très sensibles inférieur aux normes ou pratiquement inexistant. Il existe souvent de disparités considérables entre la population carcérale et la population générale, à cet égard, qui vont à l'encontre des principes relatifs aux droits de l'homme.

Les moyens de prévention, tels que les préservatifs, aiguilles et seringues stériles, désinfectants pour matériels de tatouage et informations, font souvent défaut (Onusida, 2007).

Pour Joshua et Ojong (2005), une fois condamnés et incarcérés, les détenus sont oubliés par les programmes de prévention et de traitement du VIH. Or il convient de promouvoir l'accès des détenus aux moyens de prévention-préservatifs, de produits désinfectants pour les matériels de tatouage, aiguilles et seringues stériles-conformément aux directives internationales pour la prévention du VIH dans les prisons, y compris l'accès aux médicaments de substitution pour toxicomanes (OMS, 1997). Selon Langlet (2012), il existe un faible niveau d'accessibilité aux mesures de prévention, plus particulièrement l'eau de javel, les traitements de substitution aux opiacés, les préservatifs, la prophylaxie post-exposition pour le VIH et le coiffage.

Des moyens de prévention de la transmission de la mère à l'enfant devraient être mis à la disposition des femmes enceintes en prison (Onusida, 2007). Toujours pour la même structure, les détenus devraient avoir accès à des conseils et des tests confidentiels. Aucun détenu, homme ou femme, ne devrait être pénalisé ou isolé parce qu'il est séropositif. Dans les cas avérés de sida, les malades devraient avoir accès à un traitement antirétroviral, recevoir une alimentation appropriée pour leur permettre de suivre leur traitement et avoir accès à des moyens de prévention et traitement des 
maladies opportunistes ; les maladies en phase terminale devraient également bénéficier de la mise en liberté pour des motifs humanitaires.

Selon ACT UP-Paris (2008), tous les traitements antirétroviraux sont disponibles en prison. Cependant, hors de l'entrée en détention ou lors des transferts, il arrive encore qu'il y ait des carences de quelques jours dans la prescription et l'accès aux antirétroviraux. Selon l'initiative Esther (2009), les conditions de vie des détenus encore très difficiles (promiscuité, un seul repas par jour et par détenu, mauvaise hygiène...) ne permettent pas de réduire certains comportements à risque. L'impossibilité de distribuer des préservatifs au sein de la prison et l'interdiction du dépistage chez les mineurs contribuent également à la transmission. De plus le manque de ressources humaines. A cela s'ajoute la difficulté du suivi des patients à leur sortie de prison. Tous les prisonniers séropositifs libérés sont perdus de vue malgré le dispositif de préparation et d'accueil à la sortie mis en place. Pour Dormont (1996), les conditions de vie et de prise en charge des détenus atteints par le VIH se sont beaucoup améliorées ces dernières années en raison de l'intervention des centres d'information et de soins pour l'immunodéficience humaine (CISIH) au sein des établissements pénitentiaires et de la reforme de l'organisation des soins en prison. Il n'en reste pas moins que la prise en charge des malades détenus est restée, dans certains établissements, trop largement déficiente. Pour terminer Langlet (2012) souhaiterait que l'égalité de la prise en charge sanitaire entre le milieu libre et le milieu carcéral soit enfin respectée.

Cette revue de littérature aura permis de mettre en relief la qualité des écrits réalisées sur la notion de prise en charge. Au regard des recherches effectuées dans le domaine du VIH/Sida à Abidjan, dans le cadre de cette étude et bien d'autres, il se dégage une véritable problématique dans la prise en charge des détenus infectés par le VIH/Sida. Les conditions de vie dans les prisons détériorent la prise en charge des détenus atteints du VIH/Sida. Aussi cette étude a-t-elle pour objectif d'analyser les différentes prises en charges reçues par détenus atteints du VIH/Sida à la maison d'arrêt et de correction d'Abidjan (MACA). Cette préoccupation laisse apparaitre une question à savoir, quelles sont les répercussions des différentes prises en charge sur les conditions de vie des prisonniers infectés par le VIH/Sida ?

Répondre à une telle question, revient à analyser les différentes prises en charge que bénéficient les détenus malades du Sida et leurs impacts sur leurs conditions de vie.

Ce travail de recherche tentera de vérifier l'hypothèse opératoire suivante : la prise en charge a une répercussion sur les conditions de vie des détenus infectés par le VIH/Sida. 


\section{Methodologie}

\section{-Site et participants}

Cette étude s'est déroulée à la maison d'arrêt et de correction d'Abidjan (MACA), la plus grande prison de Côte d'Ivoire. La MACA est située dans la commune de Yopougon, précisément entre le vaste quartier périphérique de Yopougon et l'ancien parc national du Banco. Elle a été construite, conformément aux standards internationaux contenus dans l'ensemble des règles relatives au traitement des détenus. En effet la MACA construite pour une capacité théorique de 1948 places. Aujourd'hui l'effectif carcéral s'élève à plus de 4424 détenus. Les enquêtes se sont effectivement déroulées sur le site de la MACA.

La population de cette étude est constituée du personnel médical et paramédical (médecin, infirmier, aide soignant, assistant social), les pairs éducateurs, les religieux, les responsables d'ONG, les gardes pénitentiaires et les détenus (infectés et sains). L'échantillon d'enquête estimé à 25 personnes reparties de manière suivante : 1 médecin, linfirmier, lassistant social, 1pasteur, limam, 1membre groupe expertise France, 2 pairs éducateurs, 3 gardes pénitentiaires, 2 responsables d'ONG, 8 détenus infectés et 4 détenus sains. Il a été constitué autour des personnes volontaires et surtout disponibles.

Les investigations ont durée un mois et demi soit 45 jours autour de la population désignée.

\section{-Instruments de recueil des données}

Au cours de l'étude, le protocole de recherche a porté sur l'étude documentaire qui a permis de faire la recension des écrits portant sur la thématique. Nous avons eu recours également au dossier carcéral et l'observation de chaque détenu interrogé. Un entretien libre individuel a été réalisé auprès des enquêtés. L'entretien libre individuel a pour avantage de permettre aux individus interrogés de développer librement leurs pensées.

\section{-Analyse des données}

Concernant l'analyse des données recueillies, nous avons opté pour l'analyse de contenu et l'analyse qualitative car il s'agit de faire ressortir le sens des discours et des réponses apportées à l'entretien.

Nous avons eu recours aussi aux récits de vie qui sont des expériences du parcours de vie des détenus infectés par le VIH/Sida. Ces expériences sont des sources d'analyse de données. Il faut aussi retenir que dans le cadre de l'élaboration de ce travail, nous avons utilisé des prénoms pour garder le caractère anonyme. 


\section{Resultats}

Les résultats obtenus dans cette étude se présentent sous trois dimensions : la sensibilisation et dépistage volontaire du VIH/Sida, les types de prises en charge bénéficiés par les détenus infectés, conditions de vie et prise en charge.

\section{Sensibilisation et dépistage volontaire du VIH/Sida}

La sensibilisation et dépistage du VIH/Sida sont des maillons essentiels de la prévention. $\mathrm{C}$ est à travers ces deux éléments que commence la prise en charge. En effet, la prison étant un nouvel environnement pour le condamné l'administration carcérale est dans l'obligation d'informer ce dernier afin qu'il s'imprègne des réalités de ce milieu (la prison). Le détenu, dès son mandat de dépôt assiste à des séances appelées séances de l'IEC (information éducation communication) dirigées par des pairs éducateurs. Séances au cours desquelles le détenu recevra des informations sur le $\mathrm{VIH} / \mathrm{Sida}$, les risques de contamination et l'intérêt de faire le test de dépistage. Cette séance se déroule dans une salle pour un entretien d'ensemble afin de parler des problèmes de santé, des règles d'hygiène, d'alimentation pour assurer un bon état de santé.

En ce qui concerne la sensibilisation, elle est dévolue aux pairs éducateurs. "Cette tache nous est assignée et nous la faisons avec amour et dévouement» raconte un pair éducateur. Il ajoute : "notre rôle est d'informer les responsables de cellules pour une activité de proximité qui consiste à développer un thème sur le VIH en vue d'amener ceux qui n'ont pas accepté de se faire dépister à le faire. Nous leur disons aussi de faire attention aux objets tranchant et coupant car cela constitue également un facteur de risque ». Au cours des séances de sensibilisation, les détenus sont exhortés au dépistage. Le test de dépistage n'est pas obligatoire. Il permet de catégoriser la population carcérale afin de prévenir les risques de contamination. Laurent, un pair éducateur dit ceci : "ici, le dépistage du VIH est volontaire, on oblige aucun détenu à faire le test. On lui montre simplement le bien fondé du dépistage ».

Ce dépistage se déroule à l'infirmerie de la MACA sous le contrôle de l'administration pénitentiaire. Et les résultats obtenus sont confidentiels. La gestion des dossiers des détenus déclarés positifs au VIH/Sida se fait dans le secret. Ce sont des codes qui identifient le détenu et le met en relation avec son médecin traitant. Le détenu dépisté positif est invité à assister à des séances de C.C.C (communication pour le changement de comportement). Au cours de ces séances, il est informé sur l'enjeu de son statut, de l'aider à accepter sa maladie, à changer de comportement et à se mettre à la disposition des médecins pour bénéficier des différents soins, dans le but d'améliorer son état de santé et surtout de ne pas disséminer la maladie. Il 
reçoit enfin des conseils allant dans le sens de la prise en charge. C'est ce que explique Jean Marc détenu infecté par le VIH/Sida en ces termes : « quand j'ai été dépisté positif, j'ai été déprimé, j'étais au bord du gouffre. Pendant les nombreuses séances de communication pour le changement de comportement auxquelles j'ai assistée, je me sens bien à présent. Je reçois une prise en charge comme les autres $》$.

Les pathologies de précarité, de promiscuité exposent le malade du sida à plusieurs infection appelées infections opportunistes qui pourraient le rende encore plus vulnérable. "Il lui serait par conséquent judicieux de connaitre son statut afin de prendre la décision adéquate pour éviter d'engager le pronostic vital » affirme un médecin de la MACA.

\section{Types de prises en charge bénéficiés par les détenus infectés par le VIH/Sida}

Il existe trois types de prises en charge que les prisonniers de la MACA reçoivent. Il s'agit de la prise thérapeutique ou médicale, la prise en charge psychologique et la prise en charge nutritionnelle ou alimentaire.

Concernant la prise en charge thérapeutique, tout commence par un bilan dit pré-thérapeutique. En effet, ce bilan détermine biologiquement la qualité fonctionnelle de tous les organes nobles à savoir le foie, le rein, le sang pour connaitre la capacité du patient à supporter les ARV (antirétroviraux). L'examen biologique prend en compte les CD4 qui sont des cellules sanguines, des cellules immunitaires spécifiques qui permettent de savoir si le malade est opérationnel au plan humanitaire. Lorsque le détenu est éligible aux antirétroviraux, des examens sont effectués pour savoir s'il est tuberculeux ou pas avant d'engager le traitement à travers l'analyse de son crachat. Si le résultat est négatif, une radiologie est faite pour être sûr. S'il est co-infecté, il sera sous traitement de co-infecté jusqu'à ce qu'il soit guérit de la tuberculose. Si non, il ne sera soumis uniquement qu'au traitement du Sida. Arsène, un détenu atteint du Sida dit ceci : "depuis que j'ai fait mes examens biologiques, mon médecin m'a mis sous traitement $A R V$. Je prend régulièrement mes médicaments et je me porte bien ».

Pour la prise en charge psychologique, elle est assurée principalement par les infirmiers spécialisés en psychiatrie, les assistants sociaux, les associatifs et certains religieux. Avec la collaboration du corps médical, certains détenus sont formés sur la question du VIH/Sida et les techniques d'approche des personnes infectées d'où l'appellation de pairs éducateurs. Ceux-ci jouent le rôle de personne intermédiaire entre le médecin traitant et détenu malade.

En effet, dès l'annonce du résultat du test, le détenu est abattu et désespéré. Face à cette situation, le malade doit être fort psychologiquement. Le faite de se savoir séropositif est un coup dur pour lui. «En plus, il est tout 
seul en prison et il n'a ni amis, ni famille, à qui confier ses peines et recevoir un minimum de réconfort moral. En prison, il est seul face à son destin. Toute sorte d'idées lui passent par la tête", nous a confié un pair éducateur."Avant la crise un détenu infecté s'est suicidé car ne pouvant pas supporter sa situation " révèle un médecin de la MACA. Afin d'éviter de tel drame, il a été confié aux pairs éducateurs, la mission d'approcher le malade, de l'écouter s'il veut se confier, de lui donner des conseils, de l'aider à surmonter sa nouvelle réalité, de le réconforter, de jouer le rôle d'un proche. Ainsi un détenu malade évoque ceci : " mon pair éducateur est pour moi ma nouvelle famille, il me donne beaucoup de conseils, sans lui, je serai perdu $»$.

Cette mission est aussi dévolue aux ONG (organisations non gouvernementales), aux religieux.

Pour la prise en charge nutritionnelle, le détenu de la MACA a droit à un seul repas par jour. Or le malade du sida doit bénéficier d'une alimentation saine et équilibrée afin de garder son poids corporel et sa forme physique. Cela lui permettra de maintenir, de renforcer son système immunitaire et de protéger son organisme contre les infections opportunistes. Comme le signifie Dr Ouattara, médecin à la MACA : "si le malade est réceptif à l'ARV, il a un très grand appétit. Il lui faut un plus que les autres ».Alain détenu vivant avec le VIH renchérit: "en mangeant uniquement que la nourriture qu'on nous donne ici, je ne fais qu'empirer mon état de santé car toutes les vitamines manquent dans cette nourriture ».Ce qui fait que l'ONG expertise France apporte son soutien à travers un appui alimentaire constitué d'un repas à midi pour tous les détenus souffrant de tuberculose de VIH/Sida et les maladies grabataires.

Aussi les détenus présentant des signes de malnutrition bénéficient-ils de biscuits et de pâtes alimentaires apportées par des ONG. Ces biscuits et pâtes alimentaires constituent un traitement pour ceux-ci. En plus, il existe au sein de la MACA, une coopérative des surveillants pénitenciers qui désignent des femmes (détenues), pour faire des repas et ensuite les vendre aux détenus qui voudraient les acheter. Selon les propos d'un médecin, certains détenus refusent de manger cette nourriture sous prétexte qu'elle est mal faite. "Si le détenu veut suivre son traitement correctement, il doit avoir le soutien financier et nutritionnel de ses parents car une nutrition complète renforce l'effet thérapeutique des médicaments » affirme un pair éducateur.

\section{Conditions de vie et insuffisance dans la prise en charge}

Les conditions de vie des détenus ont un impacte considérable sur la prise en charge des détenus vivant avec le VIH/Sida. Ainsi lors de nos investigations, nous avons constaté que les conditions de vie des détenus sont difficiles. Au niveau alimentaire, tous les détenus reçoivent officiellement un 
seul repas par jour. Ce qui ne permet pas aux prisonniers malades de suivre correctement leur traitement. D’ailleurs ce qu'évoque Martin, prisonnier vivant avec le VIH/Sida: «je reçois un repas par jour. Ce repas n'est non seulement insuffisant mais très pauvre. Ce sont mes parents qui m'envoyent à chaque fois des aliments qui me permettent de prendre mes médicaments. Il y a aussi certaines ONG de bienfaisance qui nous aident. Voila, comment j'arrive à vivre ». Pour soutenir Martin, Seydou ajoute : "la nourriture que nous recevons en prison n'est pas équilibrée. Ce qui fait que parmi nous, beaucoup de détenus souffrent de béribéri ".

La promiscuité à la MACA est une réalité. "La prison est très peuplée. Les prisonniers se retrouvent en surnombre, ici. On ne construit pas de nouveaux locaux mais chaque jour que Dieu fait, on nous envoie de nouveaux pensionnaires" relate un garde pénitentiaire. Cette situation favorise la propagation de certaines maladies contagieuses telles que la gale, tuberculose et le VIH/Sida.

Toujours à propos des conditions de vie, à la MACA, les détenus ne reçoivent ni eau de javel, ni désinfectants pour rendre propre leur environnement. Ce qui rend ce lieu insalubre. Outre ces produits, les prisonniers ne reçoivent non plus des préservatifs pour avoir des rapports sexuels protégés afin d'éviter la transmission du VIH/Sida. Il y a aussi la non distribution de seringues jetables pour les toxicomanes qui les utilisent. Sans de nouvelles seringues, les toxicomanes sont obligés d'utiliser une seringue à plusieurs et de manière répétée. Ainsi de cette façon, le sida gagne du terrain. Alors ces conditions de vie sapent la prise en charge des détenus vivant avec le VIH/Sida.

En ce qui concerne les insuffisances dans la prise en charge des détenus infectés, elles se traduisent sur le terrain par manque du personnel d'encadrement et suivi, par une limitation du dépistage aux adultes seulement, par une absence de suivi du détenu malade hors de la prison et surtout des obstacles dans la prise en charge psychologique, alimentaire et thérapeutique.

Pour attaquer ces insuffisances, Dr Ouattara, médecin à la MACA fustige le non suivi des prisonniers malades libérés ou transférés ailleurs : " aucun mesure d'accompagnement n'a vraiment été mise en place pour les prisonniers après libération, alors qu'ils sont toujours sous traitement antituberculeux. Par ailleurs, il n'est pas toujours facile d'assurer le suivi des détenus malades qui sont transférés d'un établissement à un autre ". 


\section{Discussion et conclusion}

Au regard des résultats de cette étude, nous pouvons affirmer que la prise en charge des détenus infectés par le $\mathrm{VIH} /$ Sida se situe à trois niveau à savoir : au niveau psychologique, nutritionnel et thérapeutique.

Selon Holzbauer (2016), si les antirétroviraux, les antituberculeux et antipaludéens sont livrés gratuitement au dispensaire de la prison, les autorisations d'accès à l'infirmerie relèvent des prisonniers chefs de building ou chefs d'étage, et sont soumises à des passe-droits que doivent verser les détenus malades.

Pour Blibolo cité par Ahissan (2010), les différents types de prises en charge que les personnes vivant avec le $\mathrm{VIH} /$ Sida sont à mesure de recevoir sont thérapeutiques et psychosociales. Ainsi pour lui, ces deux types de prises en charge paraissent nécessaires voire même indispensables pour que l'individu malade se sente mieux dans sa peau.

Selon la revue ACT UP-Paris (2008), tous les antirétroviraux sont disponibles en prison. Pour Langlet (2012), la prise en charge médicale des personnes séropositives s'est améliorée au fil des années. Mais les contingences organisationnelles, la crainte de la stigmatisation et les problématiques sociales des détenus compromettent parfois ces avancées. Toujours, allant dans le même sens, Guérin (2003), soutient que la prise en charge sanitaire des détenus est confiée au service public hospitalier. Ces données sont confirmées par les résultats de cette étude.

Concernant la prise en charge nutritionnelle, on constate que l'alimentaire manque de protéines et produits laitiers. Pour avoir une nourriture suffisante et équilibrée, un détenu devra acheter des suppléments, pour un coût d'environ 800 Francs par mois. Il ne pourra pas conserver les produits frais, n'ayant pas de réfrigérateur. La plupart des prisonniers n'ont pas de moyens de se nourrir correctement (Prestel, 1997).

Selon Dormont (1996), la dénutrition qui atteint, à terme, la majorité des patients, retentit sur la durée et la qualité de vie. Il convient d'y prêter une attention constante et de mettre en œuvre des stratégies pour la prévenir et la combattre. Pour Dienderé (2007), la ration de base est essentiellement composée de céréales, accompagnée plus ou moins régulièrement de sauce en moyenne deux fois par semaine (...)

Dans la plupart des maisons d'arrêt et correction, les personnes détenues reçoivent leur ration une fois par jour. Les rations alimentaires sont complétées par des achats propres ou par l'apport des familles. Egalement, des intervenants extérieurs effectuent des distributions complémentaires de nourritures sur une base régulière ou ponctuelle pour l'ensemble ou pour des groupes ciblés de détenus (indigents, mineurs...). Ces données confirment les résultats de l'enquête de terrain. 
Selon Holzbauer (2016), chargé du suivi des détenus infectés qui sortent ou qui sont transférés, Rodrigue est un conseiller à la MACA. Pour lui, nombre d'associations comme Ruban rouge auquel il appartient, travaillent à la sensibilisation des détenus. Elles dispensent des formations sur le VIH/Sida, la tuberculose, le paludisme et les MST à ceux qui se portent volontaires pour devenir des pairs éducateurs, ou, tout simplement, à ceux que cela intéresse. En effet, ces informations permettent aux détenus infectés de mieux supporter leur maladie. Les résultats de cette étude, confirment l'orientation des écrits antérieurs sur la question.

De tout ce précède, nous pouvons affirmer que les différentes formes de prises en charge (thérapeutique, psychologique et nutritionnelle) ont une incidence positive sur la qualité de vie des détenus infectés par le VIH/Sida.

\section{References:}

1. AHISSAN, K.A (2010). Le vécu de la stigmatisation par les femmes infectées par le VIH/Sida à Abidjan. Thèse de doctorat ( non publiée). UFR Criminologie, Université de Cocody Abidjan.

2. ACT UP-Paris (1999). Enquête sur le VIH en milieu carcéral. Publié en ligne : mars 1999 dans action 59.

3. ACT UP-Paris (2001). Sida, VHC et prison rapport de la mission santé/justice 2000. Publié en ligne : 29 novembre 2001 dans action 77.

4. ACT UP-Paris(2008). Le Sida en prison, un problème. Publié en ligne : juin 2008 dans action 113.

5. CURTIS, M. (2004). Fighting for prison Heath, bulletin du programme international de réduction des risques de l'open society Institute.

6. DECLARATION DE MOSCOU sur le VIH/Sida-Tuberculose dans les prisons d'Europe (2003).

7. DICTIONNAIRE Le Grand Robert de la langue Française (2005). Version2 CD-ROM.

8. DIENDERE, E. A. S. Y. (2007). Morbidité et déterminants épidémiologiques de l'infection par le virus de l'immodéficience humaine, le virus de l'hépatite $\mathrm{B}$, le tréponème pallidum et le bacille de koch en milieu carcéral de Ouagadougou-Thèse de doctorat-UFR Sciences de la santé- Université de Ouagadougou.

9. DORMONT, J. (1996). Prise en charge des personnes atteintes par le VIH. La documentation Française.

10. GUERIN, G. (2003). La santé en prison-adsp no 44 septembre 2003.

11. GOBA, B. et ZADY, C. (2017). Droit de détenus et violences en milieu carcéral en Côte d'Ivoire : le cas de la MACA. Non publié. 
12. GUICHARDAZ, O. (1999). La prise en charge sanitaire des détenus. SCERI.

13. HANZBERG, P. O. (1998). Le VIH en prison. Revue critique de l'actualité scientifique internationale sur le VIH et le virus des hépatites-no69-Automne 98.

14. HOLZBAUER, C. (2016). La prison, laboratoire de la lutte contre leVIH/Sida supplément « fonds mondial de la lutte contre le sida, la tuberculose et le paludisme ».

15. JOSHUA, L. et OJONG, M.(2005). Prisoners : the forgottens HIV/AIDS Risk group.

16. INITIATIVE ESTHER (2009). Renforcement de la prise en charge des personnes vivant avec le VIH au sein de la maison d'arrêt et de correction d'Abidjan (MACA). GIP ESTHER 62, Boulevard Garibaldi, 75015 Paris.

17. MOUQUET, M.C. et Al (1999)/ La santé à l'entrée en prison : un cumul des facteurs de risques. Etudes et Résultats : no4 janvier 1999 drees.

18. LANGLET, M. (2012). VIH en prison : les soins aux oubliettes ? Le journal du Sida no 223 avril-mai-juin 2012.

19. ONUSIDA (2006). Le point sur l'épidémie du Sida. Genève, ONUSIDA.

20. ONUSIDA (2007). Le VIH en prisons. Possibilités d'intervention en Afrique Subsaharienne. Genève, Onusida.

21. OMS (2001). La santé en prison. Genève. OMS

22. OUSMANE O. et al (2015). La vulnérabilité des détenus hommes face au VIH/Sida à Ouagadougou (Burkina Faso). La revue de tous les acteurs de la santé publique no 5, septembre-otobre 2015-P 749756

23. PRESTEL, T. (1997). Prison et Sida: la politique de l'autruche. Remaides.

24. ROTILY, M. et al (2001). Connaissances et attitudes du personnel de surveillance pénitentiaire face au VIH et/ou Sida: une enquête européenne-santé publique 2001. Volume 13, no4 PP325-328

25. ZADY, C. (2014). Relations détenus et surveillance de prison en Côte d'Ivoire. Revue internationale de Criminologie et de police technique et scientifique. Volume LXVII ; 4/14 ISSN 1424-4683. 